\title{
Expression and functions of the STAT3-SCLIP pathway in chronic myeloid leukemia cells
}

\author{
LI LI, DE ZHOU, YANLONG ZHENG and WANZHUO XIE \\ The Senior Department of Hematology, The First Affiliated Hospital of Medical College, \\ Zhejiang University, Hangzhou, Zhejiang 310006, P.R. China
}

Received July 15, 2015; Accepted September 9, 2016

DOI: $10.3892 /$ etm.2016.3768

\begin{abstract}
Chronic myeloid leukemia (CML) is a blood cell cancer with increased proliferation of granulocytes. Signal transducers and activators of transcription 3 (STAT3) is an important regulator of CML. To investigate the possible downstream factors of STAT3 and gain more insight into CML-related pathways, this study focused on the superior cervical ganglia protein 10-like protein (SCLIP, or SCG 10-like protein) and analyzed the functions of the STAT3-SCLIP pathway. The effects of STAT3 phosphorylation on SCLIP expression were examined by western blotting. Specific small interfering RNA (siRNA) was then used to knockdown SCLIP in the CML cell line K562 and the expression changes of STAT3 and factors further downstream, namely Bcl-2 and cyclin E1, were detected by RT-qPCR. Cell viability and apoptosis were also analyzed following the knockdown of SCLIP. Results showed a positive association between the phosphorylation of STAT3 and the expression of SCLIP. Knockdown of SCLIP inhibited the viability and induced the apoptosis of K562 cells. Knockdown of SCLIP did not affect the expression of STAT3 mRNA but downregulated the mRNA levels of Bcl-2 and cyclin E1. In conclusion, the results indicate that SCLIP is a direct downstream factor of STAT3, regulates $\mathrm{Bcl}-2$ and cyclin $\mathrm{E} 1$ and mediates the viability and apoptosis of CML cells. Consisting of at least these four factors, the STAT3-SCLIP pathway might play critical roles in the regulation of CML. These data provided a more profound understanding of CML-related pathways.
\end{abstract}

Correspondence to: Professor Wanzhuo Xie, The Senior Department of Hematology, The First Affiliated Hospital of Medical College, Zhejiang University, 79 Qingchun Road, Hangzhou, Zhejiang 310006, P.R. China

E-mail: xiewanzhuo2908@126.com

Key words: chronic myeloid leukemia, superior cervical ganglia 10-like protein, signal transducers and activators of transcription 3, Bcl-2, cyclin E1

\section{Introduction}

Chronic myeloid leukemia (CML) is a type of white blood cell cancer characterized by increased proliferation of granulocytes, which accounts for $20 \%$ of all leukemias in adults (1). Patients with CML usually show symptoms such as enlarged spleen and liver (2). CML is the first cancer that was found to be associated with a genetic abnormality, the Philadelphia chromosome, which results from chromosome translocation $(3,4)$. The causes of CML are difficult to identify, although some environmental stimuli, for example, exposure to ionizing radiation, appear to be risk factors (5). Imatinib and other tyrosine kinase inhibitors have been applied clinically in CML treatment strategies (6). Second generation inhibitors for imatinib-resistant patients $(7,8)$ and stem cell transplantation treatments (9) are also being investigated.

The two vital factors involved in the Philadelphia chromosome are the proto-oncogene $A B L$ and the breakpoint cluster region $B C R$. The two genes participate in the activation of several signaling pathways resulting in the altered adhesion, mitogenic activation and inhibition of apoptosis of CML cells (10). Signal transducers and activators of transcription (STAT) are important factors among these pathways, which are activated in blood cells from acute myeloid leukemia and CML (11). Among the STAT family members, STAT3 has been demonstrated to function in CML via the Janus activated kinase (JAK)/STAT pathway (12) and helps to mediate the anticancer activity of certain phytochemicals in the combination therapy of CML (13). Furthermore, its contribution to the regulation of the migration and viability of CML cells is indicated by molecular target studies $(14,15)$. However, the detailed composition of the STAT3 pathway remains unclear, particularly for the downstream factors of STAT3.

A previous study demonstrated the interactions between STAT3 and superior cervical ganglia protein 10-like protein (SCLIP, or SCG10-like protein) in the breast cancer cell line MCF-7 (16). Therefore, the present study focused on the roles of SCLIP in the CML cell line K562, with the aim of identifying the downstream factors of STAT3 to reveal the roles of this pathway in the regulation of CML. First, the relationship between STAT3 and SCLIP was analyzed. Then the knockdown of SCLIP was conducted in the CML cell line K562 using specific small interfering RNA (siRNA) and cell viability and apoptosis were compared between transfected 
and untreated cells. Also, the expression changes of two possible downstream factors of SCLIP were examined. The results should provide a more comprehensive understanding of the CML-related STAT3-SCLIP pathway.

\section{Materials and methods}

Cell culture. The leukemia model cell line K562 (Sigma-Aldrich; Merck Millipore, Darmstadt, Germany) was cultured in Roswell Park Memorial Institute-1640 medium (Sigma-Aldrich) supplemented with $2 \mathrm{mM}$ glutamine (Sigma-Aldrich), 10\% fetal bovine serum (HyClone FBS; GE Healthcare Life Sciences, Logan, UT, USA), 100 U/ml penicillin and $100 \mathrm{mg} / \mathrm{ml}$ streptomycin (Beyotime Institute of Biotechnology, Shanghai, China). The cells were maintained in a humidified atmosphere with $5 \% \mathrm{CO}_{2}$ at $37^{\circ} \mathrm{C}$. The medium was changed weekly and cells were plated at a density of $1 \times 10^{5}$ cells $/ \mathrm{ml}$. To explore the correlation between phosphorylated STAT3 (p-STAT3) and SCLIP, cells $\left(1 \times 10^{6}\right)$ were seeded onto 6-well plates. With different treatment, cells were divided into three groups: Cells without treatment, cells treated with JSI-124 ( $1 \mu \mathrm{M}$; La Jolla, CA, USA) for $24 \mathrm{~h}$ and cells treated with recombinant interleukin-21 (10 ng/ml; Sigma-Aldrich) for $24 \mathrm{~h}$.

Cell transfection. Human SCLIP-specific double-strand siRNA (SCLIP-siRNA) and the negative control siRNA were purchased from Ambion (Thermo Fisher Scientific, Inc., Waltham, MA, USA). Cell transfection was performed using Lipofectamine ${ }^{\circledR}$ RNAiMAX Reagent (Ambion) according to the manufacturer's protocol. Prior to transfection, the K562 cells were cultured and seeded to become $60 \%$ confluent in 96-well plates. The SCLIP-siRNA (1 pmol for the SCLIP-siRNA group) or the negative control siRNA (1 pmol for the siRNA control group) was diluted and added to the corresponding wells. The cells were then incubated for 1 day at room temperature. The transfection efficiency was determined using a fluorescein-labeled siRNA. Untransfected cells served as the control group.

Cell proliferation and viability assays. Cell proliferation and viability assays were conducted with Cell Proliferation kit I (Roche Life Science, Branford,CT,USA) according to the manufacturer's protocol. Specifically, cells were added to 96 -well plates at a concentration of $2 \times 10^{4}$ cells $/ \mathrm{ml}$ and cultured for 3 days. 3-(4,5-Dimethylthiazol-2-yl)-2,5-diphenyltetrazolium (MTT; $5 \mathrm{mg} / \mathrm{ml}$ ) diluted in phosphate-buffered saline (PBS) was added to each well and incubated with the cells for $4 \mathrm{~h}$. The cells were then centrifuged at $80 \mathrm{xg}$ for $10 \mathrm{~min}$ at $4^{\circ} \mathrm{C}$, collected and resuspended in dimethylsulfoxide. The plates were shaken until the crystals dissolved. The absorbance was determined at $490 \mathrm{~nm}$ using a spectrophotometer at $0 \mathrm{~h}, 72 \mathrm{~h}$ and 7 days after transfection. All assays were performed in triplicate.

Cell apoptosis assays. Cell apoptosis assays were conducted by flow cytometry (FCM) using Annexin V-fluorescein isothiocyanate (FITC)/propidium iodide (PI). An Annexin V-FITC/PI Apoptosis Detection kit (Beijing Solarbio Science $\&$ Technology Co., Ltd., Beijing, China) was used and the experiment was performed according to the manufacturer's protocol. Specifically, the cell concentration was adjusted to $5 \times 10^{5}$ cells $/ \mathrm{ml}$, and the cells were centrifuged at $200 \times \mathrm{g}$ for $5 \mathrm{~min}$ at room temperature and collected. After washing with cold PBS 3 times, the cells were resuspended in $200 \mu \mathrm{l}$ $1 \mathrm{X}$ Binding Buffer, $10 \mu \mathrm{l}$ Annexin V-FITC and $10 \mu \mathrm{l} \mathrm{PI}$ and then incubated in the dark for $15 \mathrm{~min}$ at room temperature. Prior to detection by FCM, another $300 \mu 1$ 1X Binding Buffer was added. Detection was performed at $0 \mathrm{~h}, 72 \mathrm{~h}$ and 7 days after transfection. The Annexin V-positive and PI negative cells located in quadrant $3(\mathrm{Q} 3)$ of the plot were considered to be apoptotic cells and were used for comparison among groups.

Reverse transcription-quantitative polymerase chain reaction $(R T-q P C R)$. The expression levels of various mRNAs were detected by RT-qPCR using the following primers: STAT3, forward: 5'-GCCAGAGAGCCAGGAGCA-3' and reverse: 5'-TGA AGCTGACCCAGGTAGCGCTGC-3'; SCLIP, forward: 5'-AGGAGTTATCTGTGCTGTCGC-3' and reverse: 5'-TGGTAGATGGTGTTCGGGTG-3'; $B c l$-2, forward: 5'-AAGAGCAGACGGATGGAAAAAGG-3' and reverse: 5'-GGGCAAAGAAATGCAAGTGAATG-3'; and cyclin E1, forward: 5'-GCAAGCCTCGGATTATTGCA-3' and reverse: 5'-CCTCTCTATTTGCCCAGCTCAGTA-3'. $G A P D H$ was used as the internal control, with the following primer sequences: Forward: 5'-GAGTCAACGGATTTGGTC GT-3' and reverse: 5'-GACAAGCTTCCCGTTCTCAG-3' The total RNA of cells was isolated with TRIzol (Invitrogen; Thermo Fisher Scientific, Inc.) as well as DNase I (Promega Corporation, Madison, WI, USA) and complementary DNA synthesis was performed using a PrimerScript RT Reagent kit (Takara Biotechnology Co., Ltd., Dalian, China) according to the manufacturer's protocol. qPCR was conducted in a 20- $\mu 1$ reaction system using a LightCycler 96 Real-Time PCR System (Roche Life Science, Basel, Switzerland) with the following cycling procedures: Pre-denaturation at $94^{\circ} \mathrm{C}$ for $2 \mathrm{~min}$, followed by 40 cycles comprising denaturation at $94^{\circ} \mathrm{C}$ for $1 \mathrm{~min}$, annealing at $60^{\circ} \mathrm{C}$ for $1 \mathrm{~min}$ and extension at $72^{\circ} \mathrm{C}$ for $1 \mathrm{~min}$, and then a final extension step at $72^{\circ} \mathrm{C}$ for $7 \mathrm{~min}$. All the experiments were performed in triplicate. The data were calculated using the $2^{-\Delta \Delta \mathrm{Cq}}$ method (17).

Western blot analysis. The cells were harvested and washed twice with cold PBS. Protein samples were extracted with cell lysis buffer containing $2 \%$ Nonidet P-40, $0.5 \%$ sodium deoxycholate, $0.1 \%$ sodium dodecyl sulfate (SDS), $100 \mu \mathrm{g} / \mathrm{ml}$ phenylmethylsulfonyl fluoride and $10 \mu \mathrm{g} / \mathrm{ml}$ leupeptin. Equal amounts of protein $(20 \mu \mathrm{g})$ from each group were separated by $12 \%$ SDS-polyacrylamide gel electrophoresis and transferred to a polyvinylidene fluoride membrane. The blot was blocked with 5\% skimmed milk in Tris-buffered saline Tween-20 (TBST) buffer (pH 8.0) overnight at $4^{\circ} \mathrm{C}$ and then incubated with specific primary antibodies for phosphorylated (p)-STAT3 (1:500; cat. no. sc-135649), SCLIP (1:500; cat. no. sc-85907) or $\beta$-actin $(1: 1,000$; cat. no. sc-130656) (Santa Cruz Biotechnology, Inc., Dallas, TX, USA) for $2 \mathrm{~h}$ at room temperature. The membrane was then washed with TBST and incubated with horseradish peroxidase-conjugated goat anti-rabit $\operatorname{IgG}(1: 5,000$; cat. no. sc-2004) and donkey anti-goat 
A

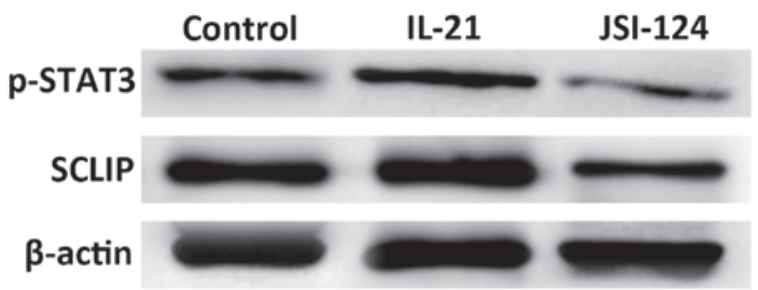

B

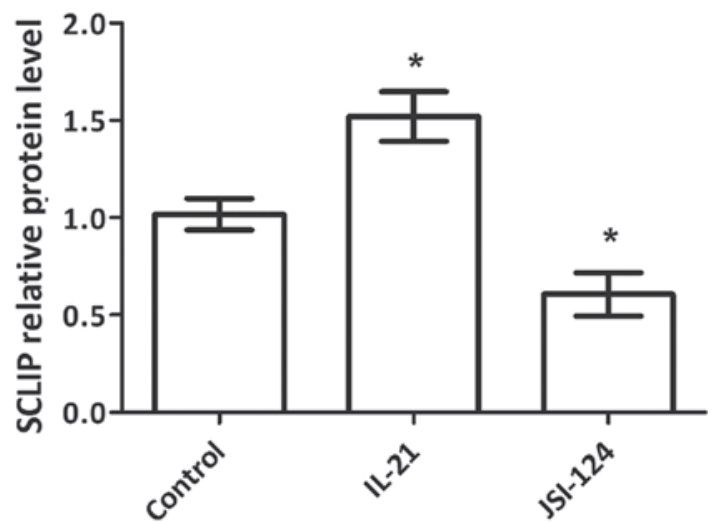

Figure 1. Association between the expression levels of p-STAT3 and SCLIP. (A) Western blotting results showing the expression changes of SCLIP after the phosphorylation of STAT3 was promoted by IL-21 or inhibited by JSI-124. $\beta$-actin was used as an internal reference. (B) Bar graph showing the relative SCLIP protein levels determined from the western blotting results. ${ }^{*} \mathrm{P}<0.05$ vs. the control group. STAT3, signal transducers and activators of transcription 3; p-STAT3, phosphorylated STAT3; SCLIP, superior cervical ganglia protein 10-like protein; IL-21, interleukin-21; JSI-124, cucurbitacin I.

$\operatorname{IgG}(1: 5,000$; cat. no. sc-2020) secondary antibodies (Santa Cruz Biotechnology, Inc.) for $2 \mathrm{~h}$ at room temperature. Positive signals were detected using an enhanced chemiluminescence reagent (Amersham ECL Detection kit; GE Healthcare Life Sciences) and then analyzed using a Kodak Digital Imaging System (DC120; Kodak, Rochester, NY, USA). $\beta$-actin was used as the internal reference.

Statistical analysis. Differences between groups were examined using the unpaired Student's t-test. Data are presented as the mean \pm standard deviation of three independent experiements. $\mathrm{P}<0.05$ was considered to indicate a statistically significant difference. All data analyses were performed using Statistical Package for Social Sciences (SPSS) version 19 software (IBM SPSS, Armonk, NY, USA).

\section{Results}

SCLIP is a downstream molecule of STAT3. The phosphorylation levels of STAT3 and the protein expression levels of SCLIP were detected by western blotting. The results showed a positive correlation between the expression of phosphorylated STAT3 (p-STAT3) and SCLIP (Fig. 1A and B): When the phosphorylation of STAT3 was promoted by IL-21, the expression of SCLIP was upregulated and when the phosphorylation of STAT3 was inhibited by JSI-124, the expression of SCLIP was downregulated. The differences in the expression levels of SCLIP in the two treatment groups compared with those in the
A

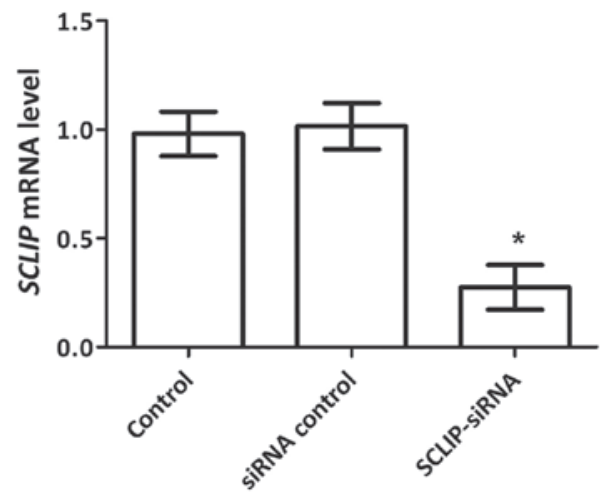

B

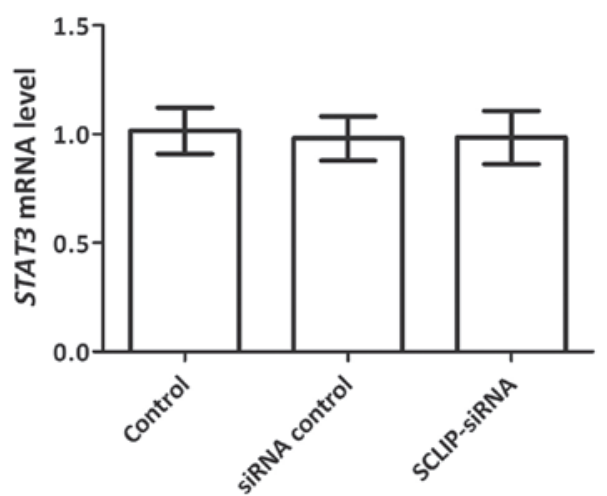

Figure 2. Relative expression levels of SCLIP and STAT3 mRNA. mRNA expression levels of (A) SCLIP and (B) STAT3 in the control group and transfected groups. ${ }^{*} \mathrm{P}<0.05$ vs. the control group. SCLIP, superior cervical ganglia protein 10-like protein; siRNA, small interfering RNA; STAT3, signal transducers and activators of transcription 3.

control group were significant $(\mathrm{P}<0.05)$. Since the expression of SCLIP could be activated by p-STAT3, these results imply that SCLIP might be a downstream molecule of STAT3.

Following the transfection of the cells with the SCLIP-specific siRNA or negative control siRNA, the mRNA expression levels of SCLIP and STAT3 in each group were detected by RT-qPCR. The expression levels of SCLIP and STAT3 mRNA in the siRNA control group were similar to those in the untransfected control group (Fig. 2). In the SCLIP-siRNA group, the expression of SCLIP mRNA was significantly downregulated $(\mathrm{P}<0.05)$ compared with that in the control group indicating that effective knockdown of SCLIP. However, the expression of STAT3 mRNA was hardly changed as SCLIP was inhibited, which implies that STAT3 is located upstream of SCLIP. These results together with the previous western blotting results, indicate that SCLIP may be upregulated by the phosphorylation of its upstream molecule, STAT3.

Knockdown of SCLIP inhibits cell viability and promotes apoptosis. The effects of SCLIP on K562 cell viability and apoptosis were tested by MTT and cell apoptosis assays, respectively. The cell viability of the SCLIP-siRNA group was inhibited and exhibited a significant difference from the control group at 7 days after transfection $(\mathrm{P}<0.05$; Fig. 3$)$. Similarly, the apoptotic cell ratio in the SCLIP-siRNA group was significantly increased at both $72 \mathrm{~h}$ and 7 days after transfection compared with that in the control group $(\mathrm{P}<0.05)$, 


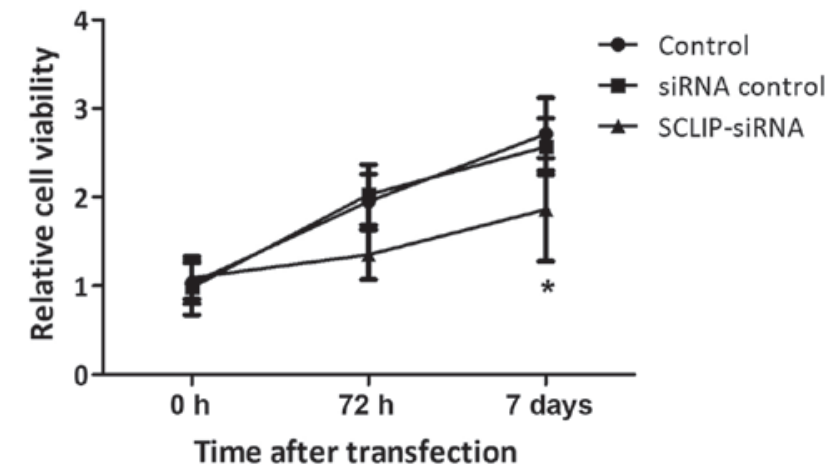

Figure 3. Relative cell viability changes after the knockdown of SCLIP. Cell viability was detected at $0 \mathrm{~h}, 72 \mathrm{~h}$ and 7 days after transfection with the negative control siRNA and the SCLIP-specific siRNA. A significant difference was found at 7 days after transfection with SCLIP-specific siRNA compared with the control group ( $\left.{ }^{*} \mathrm{P}<0.05\right)$. siRNA, small interfering RNA; SCLIP, superior cervical ganglia protein 10 -like protein.

increasing from 4.28 to $5.85 \%$ and from 4.88 to $7.14 \%$, respectively (Fig. 4). These data indicate that SCLIP was involved in the promotion of cell viability and inhibition of cell apoptosis in the K562 cells, implicating SCLIP as a regulator of the viability and apoptosis of CML cells.

Knockdown of SCLIP regulates the expression of $\mathrm{Bcl}-2$ and cyclin E1. The possible regulatory mechanisms of SCLIP in CML were studied by analyzing the changes in the expression of two anti-apoptosis genes, namely, Bcl-2 and cyclin E1, following the knockdown of SCLIP. The RT-qPCR results showed that the expression patterns of the two genes were positively associated with the expression of SCLIP (Fig. 5). That is, with the knockdown of SCLIP, both Bcl-2 and cyclin E1 mRNA expression levels were downregulated, exhibiting significant differences compared with the control group $(\mathrm{P}<0.05)$. Therefore, $\mathrm{Bcl}-2$ and cyclin $\mathrm{E} 1$ are two possible downstream factors of SCLIP. It may be hypothesized that the two factors, together with STAT3 and SCLIP, constitute a part of the signaling pathway that regulates the viability and apoptosis of CML cells.

\section{Discussion}

In this study, SCLIP is demonstrated to be a downstream factor of STAT3, since its expression is activated by the phosphorylation of STAT3, while the knockdown of SCLIP has no effect on the expression of STAT3 mRNA. In addition, SCLIP knockdown is demonstrated to inhibit the viability and promote the apoptosis of K562 cells. Two anti-apoptosis factors, Bcl-2 and cyclin E1, were observed to be downregulated when SCLIP was knocked down, indicating they may be the downstream factors of SCLIP. Therefore, the data in the present study suggest that the STAT3-SCLIP pathway consisting of these four factors (SCLIP, STAT3, Bcl-2 and cyclin E1) is involved in regulating the viability and apoptosis of CML cells.

This study presents a relatively detailed delineation of the STAT3-SCLIP pathway involving STAT3, SCLIP, Bcl-2 and cyclin E1. Since IL-21 can bind to its receptor and phosphorylate STAT3 to initiate the transcription of regulated genes (18), while cucurbitacin I (JSI-124) is able to reduce the phosphorylation level of STAT3 (19), these two factors were used in the present study to regulate the phosphorylation of STAT3. The results showed that the expression of SCLIP was regulated by p-STAT3 levels and the knockdown of SCLIP did not affect the expression of STAT3. Together with previous studies showing that STAT3 regulates and even interacts directly with SCLIP $(16,20)$, it could be inferred that SCLIP is a direct downstream factor of STAT3 in K562 cells. The presence of a direct regulatory association between SCLIP and Bcl-2 or cyclin E1 is unclear because there was no explicit evidence ruling out other possible factors between the two cascades, although microarray analyses have found that STAT3 is able to regulate the expression of Bcl-2 $(21,22)$. Therefore, it could be deduced from the existing results that Bcl-2 and cyclin E1 are located downstream of SCLIP. Considering the aforementioned data together, the basic part of this pathway is hypothesized to involve direct regulation by STAT3 of its downstream SCLIP, and two factors further downstream, Bcl-2 and cyclin E1, that are regulated by SCLIP.

SCLIP was demonstrated to promote the viability and inhibit the apoptosis of K562 cells in the present study, implying its role in the regulation of CML. Similar functions of SCLIP have been demonstrated in other cell lines and diseases. For example, SCLIP has been shown to facilitate the proliferation, invasion and migration of non-small cell lung cancer cells (23). It also promotes neurite growth by pheochromacytoma PC12 cells (24). Similarly, Bcl-2 and cyclin E1 have been demonstrated to be anti-apoptosis factors; Bcl-2 knockout or the overexpression of factors targeting Bcl-2 causes the acceleration of cell apoptosis $(25,26)$. The abnormal expression of cyclin E1 contributes to tumorigenesis (27) and its knockdown induces apoptosis in cancer cells (28). In the present study, these two anti-apoptosis factors were downregulated along with the knockdown of SCLIP, indicating their consistent roles in the regulation of the STAT3-SCLIP pathway. Therefore, generally, the activation of the STAT3-SCLIP pathway, including the upregulation of STAT3, SCLIP, Bcl-2 or cyclin E1, might result in the promotion of cell viability and inhibition of cell apoptosis. These key factors in this pathway could serve as promising molecular targets of CML treatment.

Since CML is regulated by multiple signaling pathways (10), there is a great possibility that the signaling pathway that is partly defined in the present study is connected to other important factors, thus constituting a complex regulatory network. As already mentioned, STAT3 is a member of the JAK/STAT pathway that regulates CML (12). It also participates in the phosphatidylinositol 3-kinase/mammalian target of rapamycin/STAT3 pathway to regulate cell viability in breast cancer stem-like cells (29). Bcl-2 is involved in pathways, such as the extracellular signaling regulated kinase pathway and the nuclear factor $\kappa \mathrm{B} / \mathrm{Bcl}-2$ pathway, that regulate cancer cells $(30,31)$. Furthermore, the cyclin E1-Cdk 2 complex is the endpoint of several pathways in the growth control of cancer cells (32). It may be deduced that CML is regulated by a complex pathway network involving STAT3, SCLIP, Bcl-2 and cyclin E1. Therefore, a comprehensive understanding of the regulatory mechanisms is of great significance.

In summary, this study demonstrates that SCLIP, as a direct downstream factor of STAT3, is able to regulate the 
A

Control

$\mathrm{Oh}$

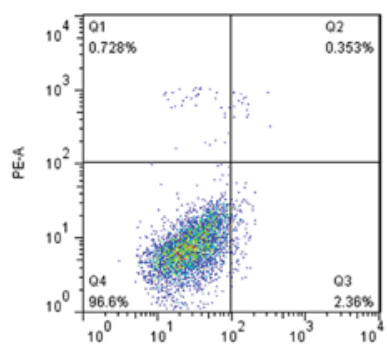

$72 \mathrm{~h}$

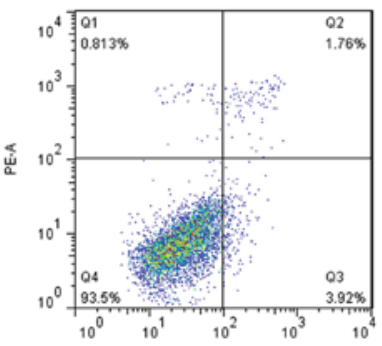

FITC-A

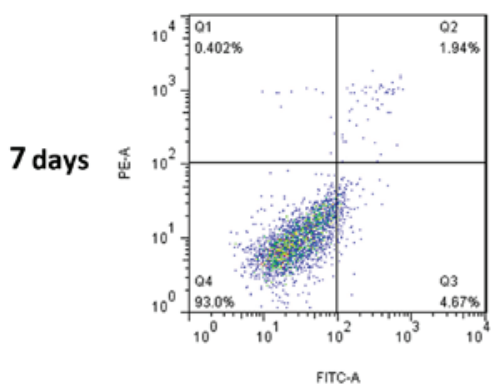

SiRNA control
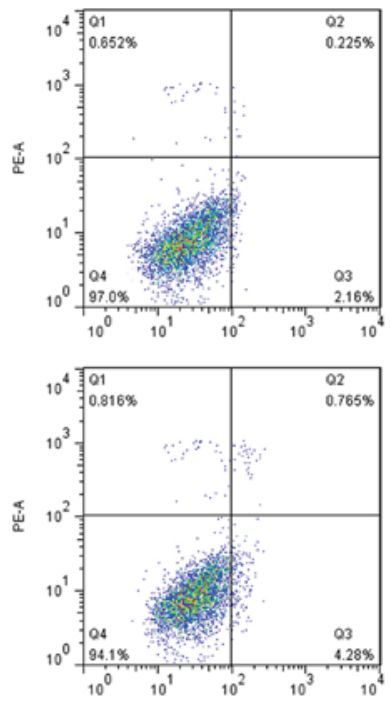

FITC-A

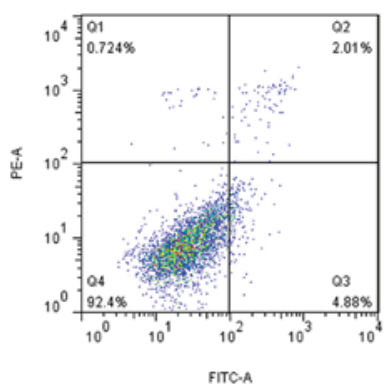

SCLIP-siRNA
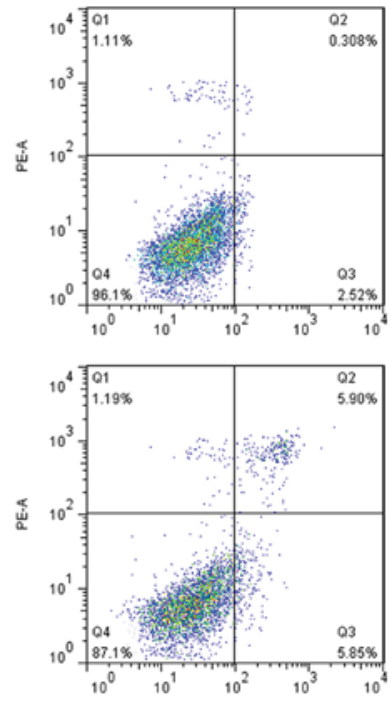

FITC-A

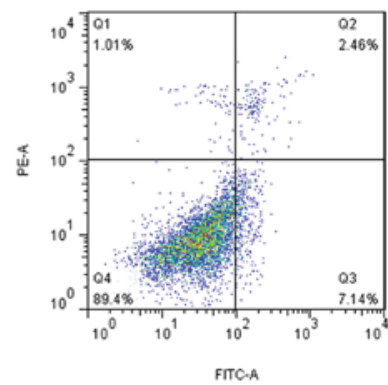

B

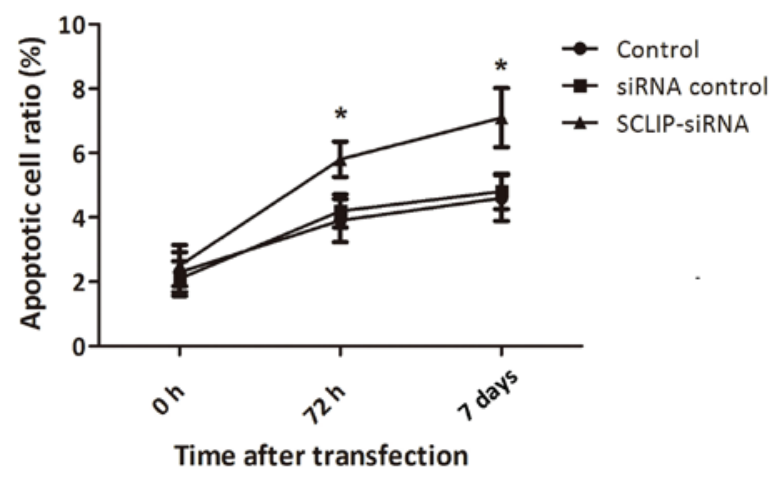

Figure 4. Changes in apoptotic cell ratios following the knockdown of SCLIP. (A) Flow cytometry results showing different cell groups of the control group, the siRNA control group and the SCLIP-siRNA group at $0 \mathrm{~h}, 72 \mathrm{~h}$ and 7 days after transfection. The horizontal axis and the vertical axis indicate the relative signal intensities of Annexin V and propidium iodide, respectively. (B) Apoptotic cell ratios based on the cell ratios in Q3. siRNA, small interfering RNA; SCLIP, superior cervical ganglia protein 10-like protein; Q1-Q4, quadrant 1-quadrant 4; FITC, fluorescein isothiocyanate; PE, phycoerythrin. " P $<0.05$ vs. the control group.

A

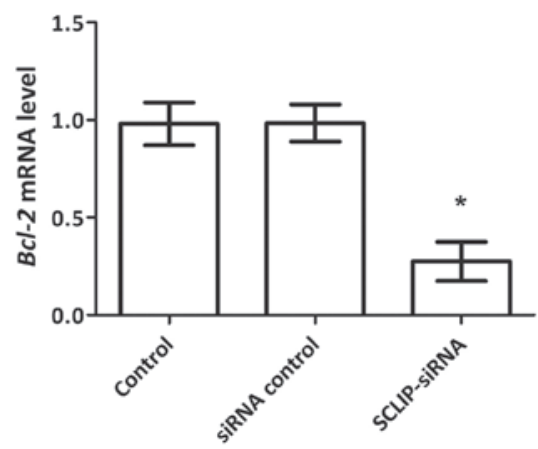

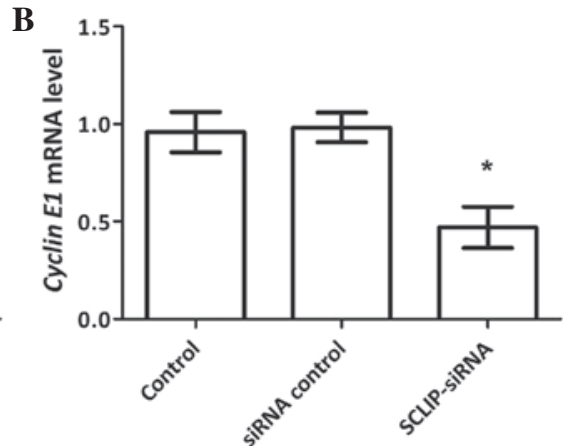

Figure 5. Expression changes of Bcl-2 and cyclin E1 mRNA following the knockdown of SCLIP. Changes in the levels of (A) Bcl-2 and (B) cyclin E1 mRNA following transfection. siRNA, small interfering RNA; SCLIP, superior cervical ganglia protein 10 -like protein. * $\mathrm{P}<0.05$ vs. the control group. 
viability and apoptosis of CML cells. The STAT3-SCLIP pathway, a basic pathway model for CML regulation is described, including the direct interaction between STAT3 and SCLIP, and factors further downstream, Bcl-2 and cyclin E1. These data facilitate future molecular studies of CML regulation and suggest alternative molecular targets for CML treatment. A more profound understanding of the associated signaling pathway network is necessary for further clinical research.

\section{References}

1. Stein B and Smith BD: Treatment options for patients with chronic myeloid leukemia who are resistant to or unable to tolerate imatinib. Clinical Therapeutics 32: 804-820, 2010.

2. Chaudhary V, Sachdeva P, Karanth P and Arora R: Spontaneous hemoperitoneum in chronic myeloid leukemia: An unusual etiology. J Hematol (Brossard) 2: 40-41, 2013.

3. Faderl S, Talpaz M, Estrov Z and Kantarjian HM: Chronic myelogenous leukemia: Biology and therapy. Ann Intern Med 131: 207-219, 1999.

4. Hehlmann R, Hochhaus A and Baccarani M; European LeukemiaNet: Chronic myeloid leukaemia. Lancet 370: 342-350, 2007.

5. Kabel AM and Elmaaboud MAA: Cancer: Role of nutrition, pathogenesis, diagnosis and management. World Journal of Nutrition and Health 2: 48-51, 2014

6. Shah NP, Guilhot F, Cortes JE, Schiffer CA, le Coutre P, Brümmendorf TH, Kantarjian HM, Hochhaus A, Rousselot P, Mohamed $\mathrm{H}$, et al: Long-term outcome with dasatinib after imatinib failure in chronic-phase chronic myeloid leukemia: Follow-up of a phase 3 study. Blood 123: 2317-2324, 2014.

7. Bixby D and Talpaz M: Seeking the causes and solutions to imatinib-resistance in chronic myeloid leukemia. Leukemia 25 : 7-22, 2011.

8. Weisberg E, Manley PW, Cowan-Jacob SW, Hochhaus A and Griffin JD: Second generation inhibitors of BCR-ABL for the treatment of imatinib-resistant chronic myeloid leukaemia. Nat Rev Cancer 7: 345-356, 2007.

9. Pavlů J and Apperley JF: Allogeneic stem cell transplantation for chronic myeloid leukemia. Curr Hematol Malig Rep 8: 43-51, 2013.

10. Deininger MW, Goldman JM and Melo JV: The molecular biology of chronic myeloid leukemia. Blood 96: 3343-3356, 2000.

11. Chai SK, Nichols GL and Rothman P: Constitutive activation of JAKs and STATs in BCR-Abl-expressing cell lines and peripheral blood cells derived from leukemic patients. J Immunol 159: 4720-4728, 1997.

12. Zhu JF, LI ZI, Zhang GS, Meng K, Kuang WY, Li J, Zhou XF, LI RJ, Peng HI, Dai CW, et al: Icaritin shows potent anti-leukemia activity on chronic myeloid leukemia in vitro and in vivo by regulating MAPK/ERK/JNK and JAK2/STAT3/AKT signalings. PLoS One 6: e23720, 2011.

13. Jung JH, Kwon TR, Jeong SJ, Kim EO, Sohn EJ, Yun M and Kim SH: Apoptosis induced by tanshinone IIA and cryptotanshinone is mediated by distinct JAK/STAT3/5 and SHP1/2 signaling in chronic myeloid leukemia K562 cells. Evid Based Complement Alternat Med 2013: 805639, 2013.

14. Kiper HD, Tezcanli Kaymaz B, Gokbulut AA, Selvi N, Avci CB, Kosova B, Iskender G, Yandim MK, Gunduz C, Sahin F, et al: STAT pathway in the regulation of zoledronic acid-induced apoptosis in chronic myeloid leukemia cells. Biomed Pharmacother 67: 527-532, 2013.
15. Kaymaz BT, Cetintas VB, Aktan C and Kosova B: MicroRNA-520a-5p displays a therapeutic effect upon chronic myelogenous leukemia cells by targeting STAT3 and enhances the anticarcinogenic role of capsaicin. Tumour Biol 35: 8733-8742, 2014.

16. Ng DC, Lim CP, Lin BH, Zhang T and Cao X: SCG10-like protein (SCLIP) is a STAT3-interacting protein involved in maintaining epithelial morphology in MCF-7 breast cancer cells. Biochem J 425: 95-105, 2009.

17. Livak KJ and Schmittgen TD: Analysis of relative gene expression data using real-time quantitative PCR and the 2(-Delta Delta C(T)) Method. Methods 25: 402-408, 2001.

18. Zhu M, Pleasic-Williams S, Lin TH, Wunderlich DA, Cheng JB and Masferrer JL: pSTAT3: A target biomarker to study the pharmacology of the anti-IL-21R antibody ATR-107 in human whole blood. J Transl Med 11: 65, 2013.

19. Qi J, Xia G, Huang CR, Wang JX and Zhang J: JSI-124 (Cucurbitacin I) inhibits tumor angiogenesis of human breast cancer through reduction of STAT3 phosphorylation. Am J Chin Med 43: 337-347, 2015.

20. Zhang Y, Ni S, Huang B, Wang L, Zhang X, Li X, Wang H, Liu S, Hao A and Li X: Overexpression of SCLIP promotes growth and motility in glioblastoma cells. Cancer Biol Ther 16: 97-105, 2015.

21. Gritsko T, Williams A, Turkson J, Kaneko S, Bowman T, Huang M, Nam S, Eweis I, Diaz N, Sullivan D, et al: Persistent activation of stat 3 signaling induces survivin gene expression and confers resistance to apoptosis in human breast cancer cells. Clin Cancer Res 12: 11-19, 2006.

22. Zhang D, He D, Xue Y, Wang R, Wu K, Xie H, Zeng J, Wang X, Zhau HE, Chung LW, et al: PrLZ protects prostate cancer cells from apoptosis induced by androgen deprivation via the activation of Stat3/Bcl-2 pathway. Cancer Res 71: 2193-2202, 2011.

23. Nair S, Bora-Singhal N, Perumal D and Chellappan S: Nicotine-mediated invasion and migration of non-small cell lung carcinoma cells by modulating STMN3 and GSPT1 genes in an ID1-dependent manner. Mol Cancer 13: 173, 2014.

24. Kang SW, Shin YJ, Shim YJ, Jeong SY, Park IS and Min BH: Clusterin interacts with SCLIP (SCG10-like protein) and promotes neurite outgrowth of PC12 cells. Exp Cell Res 309: 305-315, 2005.

25. van Delft MF, Wei AH, Mason KD, Vandenberg CJ, Chen L, Czabotar PE, Willis SN, Scott CL, Day CL, Cory S, et al: The BH3 mimetic ABT-737 targets selective Bcl-2 proteins and efficiently induces apoptosis via Bak/Bax if Mcl-1 is neutralized. Cancer Cell 10: 389-399, 2006.

26. Wang Y, Li M, Zang W, Ma Y, Wang N, Li P, Wang T and Zhao G: MiR-429 up-regulation induces apoptosis and suppresses invasion by targeting Bcl-2 and SP-1 in esophageal carcinoma. Cell Oncol (Dordr) 36: 385-394, 2013.

27. Spruck CH, Won KA and Reed SI: Deregulated cyclin E induces chromosome instability. Nature 401: 297-300, 1999.

28. Gurzov EN and Izquierdo M: Cyclin E1 knockdown induces apoptosis in cancer cells. Neurol Res 28: 493-499, 2006.

29. Zhou J, Wulfkuhle J, Zhang H, Gu P, Yang Y, Deng J, Margolick JB, Liotta LA, Petricoin E III and Zhang Y: Activation of the PTEN/mTOR/STAT3 pathway in breast cancer stem-like cells is required for viability and maintenance. Proc Natl Acad Sci USA 104: 16158-16163, 2007.

30. Galante JM, Mortenson MM, Bowles TL, Virudachalam S and Bold RJ: ERK/BCL-2 pathway in the resistance of pancreatic cancer to anoikis. J Surg Res 152: 18-25, 2009.

31. Buchholz TA, Garg AK, Chakravarti N, Aggarwal BB, Esteva FJ, Kuerer HM, Singletary SE, Hortobagyi GN, Pusztai L, Cristofanilli $\mathrm{M}$ and Sahin AA: The nuclear transcription factor kappaB/bcl-2 pathway correlates with pathologic complete response to doxorubicin-based neoadjuvant chemotherapy in human breast cancer. Clin Cancer Res 11: 8398-8402, 2005.

32. Möröy T and Geisen C: Cyclin E. Int J Biochem Cell Biol 36: 1424-1439, 2004 